\title{
K2-30 b and K2-34 b: Two inflated hot Jupiters around solar-type stars ${ }^{\star}$
}

\author{
J. Lillo-Box ${ }^{1,2}$, O. Demangeon ${ }^{3}$, A. Santerne ${ }^{4,3}$, S. C. C. Barros ${ }^{4}$, D. Barrado ${ }^{2}$, G. Hébrard ${ }^{5,6}$, H. P. Osborn ${ }^{7}$, \\ D. J. Armstrong ${ }^{7}, 12$, J.-M. Almenara ${ }^{8,9}$, I. Boisse ${ }^{3}$, F. Bouchy ${ }^{3,10}$, D. J. A. Brown ${ }^{7}$, B. Courcol ${ }^{7}$, M. Deleuil ${ }^{3}$, \\ E. Delgado Mena ${ }^{4}$, R. F. Díaz ${ }^{7}$, J. Kirk ${ }^{7}$, K. W. F. Lam ${ }^{7}$, J. McCormac ${ }^{7}$, D. Pollacco ${ }^{7}$, A. Rajpurohit ${ }^{3,11}$, J. Rey ${ }^{10}$, \\ N. C. Santos ${ }^{4,13}$, S. G. Sousa ${ }^{4}$, M. Tsantaki ${ }^{4}$, and P. A. Wilson ${ }^{5}$ \\ 1 European Southern Observatory (ESO), Alonso de Cordova 3107, Vitacura, Casilla 19001, Santiago de Chile, Chile \\ e-mail: jlillobox@eso.org \\ 2 Depto. de Astrofísica, Centro de Astrobiología (CSIC-INTA), ESAC campus, 28692 Villanueva de la Cañada, Madrid, Spain \\ Aix Marseille Université, CNRS, Laboratoire d'Astrophysique de Marseille UMR 7326, 13388 Marseille, France \\ ${ }^{4}$ Instituto de Astrofísica e Ciências do Espaço, Universidade do Porto, CAUP, Rua das Estrelas, 4150-762 Porto, Portugal \\ 5 Institut d'Astrophysique de Paris, UMR7095 CNRS, Université Pierre \& Marie Curie, 98bis boulevard Arago, 75014 Paris, France \\ Observatoire de Haute-Provence, Université d'Aix-Marseille \& CNRS, 04870 Saint-Michel l'Observatoire, France \\ Department of Physics, University of Warwick, Gibbet Hill Road, Coventry, CV4 7AL, UK \\ Université Grenoble Alpes, IPAG, 38000 Grenoble, France \\ CNRS, IPAG, 38000 Grenoble, France \\ 10 Observatoire Astronomique de l'Université de Genève, 51 chemin des Maillettes, 1290 Versoix, Switzerland \\ 11 Astronomy and Astrophysics Division, Physical Research Laboratory, 380009 Ahmedabad, India \\ 12 ARC, School of Mathematics \& Physics, Queen's University Belfast, University Road, Belfast BT7 1NN, UK \\ 13 Depto. de Física e Astronomia, Faculdade de Ciências, Universidade do Porto, Rua Campo Alegre, 4169-007 Porto, Portugal
}

Received 28 January 2016 / Accepted 20 May 2016

\begin{abstract}
We report the discovery of the two hot Jupiters K2-30 b and K2-34b. The two planets were detected during campaigns 4 and 5 of the extension of the Kepler mission, K2; they transit their main-sequence stars with periods of $\sim 4.099$ and $\sim 2.996$ days. Subsequent ground-based radial velocity follow-up with SOPHIE, HARPS-N, and CAFE established the planetary nature of the transiting objects. We analyzed the transit signal, radial velocity, and spectral energy distributions of the two systems to characterize their properties. Both planets (K2-30 b and K2-34 b) are bloated hot Jupiters ( $1.20 R_{\text {Jup }}$ and $\left.1.22 R_{\text {Jup }}\right)$ around relatively bright $(V=13.5$ and $V=11.5)$ slow rotating main-sequence (G8 and F9) stars. Thus, these systems are good candidates for detecting the Rossiter-MacLaughlin effect in order to measure their obliquity and for atmospheric studies.
\end{abstract}

Key words. planets and satellites: detection - planets and satellites: gaseous planets - techniques: radial velocities techniques: photometric - techniques: high angular resolution

\section{Introduction}

The extension of the Kepler mission (K2; Howell et al. 2014) is photometrically monitoring different fields along the ecliptic over $\sim 80$-day time spans. Despite the shorter time span and the slightly lower photometric precision with respect to the prime mission, several tens of extrasolar planets have been detected and characterized so far. These planets cover a wide range of properties, from disintegrating Neptune-sized objects (Sanchis-Ojeda et al. 2015) through validated Earth-sized planets (e.g., Crossfield et al. 2015; Petigura et al. 2015) to resonant multi-planetary systems (Armstrong et al. 2015b; Barros et al. 2015).

Several works have provided planet candidates based on independent analysis of the light curves (e.g., Foreman-Mackey et al. 2015). However, as in the prime part of

* Full Tables 1 and 2 are only available at the CDS via anonymous ftp to cdsarc.u-strasbg. fr (130.79.128.5) or via http://cdsarc.u-strasbg.fr/viz-bin/qcat?J/A+A/594/A50 the mission, any candidate requires follow-up observations to unveil its nature. Owing to the large Kepler pixel size (around $4 \times 4$ arcsec), contaminant sources can lie within the photometric aperture. Several high spatial resolution follow-up surveys were carried out for the prime mission (e.g., Lillo-Box et al. 2012, 2014b; Adams et al. 2012; Law et al. 2014) concluding that $20-40 \%$ of the candidates have stellar companions closer than 3 arcsec. Thus, both high-resolution images and radial velocity (RV) data are needed.

The currently known extrasolar planets show an interesting population of close-in $(a<0.1 \mathrm{au})$ gaseous planets, known as hot Jupiters (HJ), with a maximum frequency at a 5-day period (Santerne et al. 2016). The detection and full characterization of these systems has become crucial to understanding early migration processes and also planet-star and planet-planet interactions. These are the best targets to study these processes because their consequences are easily detectable from the ground. For instance, HJs were found to be mostly solitary (Steffen et al. 2012), with no other planets in the system. This was explained 
Table 1. Normalized detrended K2 light curve of K2-30 (see Sect. 2.1).

\begin{tabular}{rcc}
\hline \hline BJD-2 400 000 (days) & Flux & Flux unc \\
\hline 2457067.684383 & 1.000706 & 0.000103 \\
2457067.704815 & 0.999879 & 0.000103 \\
2457067.725247 & 0.999752 & 0.000103 \\
2457067.745679 & 0.999817 & 0.000103 \\
2457067.766111 & 0.999762 & 0.000103 \\
$\ldots$ & $\ldots$ & $\ldots$ \\
\hline
\end{tabular}

Notes. The complete version of this table is available at the CDS.

by the possible suppression of rocky planet formation owing to the inward migration of the HJ early in the evolution of the system (e.g., Armitage 2003). However, the detection of inner and outer planets to the HJ WASP-47 b using K2 (Becker et al. 2015) has challenged this scenario. Also, measuring the spinorbit angle provides hints about the migration history of the system (Morton \& Johnson 2011) and this angle has already been determined for many HJs (e.g., Winn et al. 2005). Additionally, many HJs are found to be bloated. The source of this inflation is still not well understood; several mechanisms have been proposed (e.g., Batygin \& Stevenson 2010; Showman \& Guillot 2002), but none can explain the inflation by itself. In addition, transiting HJs currently represent the best chance to study exoplanet atmospheres with high-precision light curves, which allow phase curve studies.

Hence, a full characterization of a large population of HJs is necessary to analyze the migration and formation history of these systems and to study the planet-planet and planet-star interactions. We have used data from different facilities to identify and characterize the extrasolar planets K2-34 b and K2-30 b, two inflated hot Jupiters around solar-like stars. In this paper we detail the observations, data reduction, analysis, and conclusions regarding these systems.

\section{Observations and data handling}

\subsection{K2 photometry}

The star K2-30 (EPIC210957318, 03:29:22.07 +22:17:57.9) was observed by K2 during its campaign 4, between February 7 th and April 23rd, 2015. K2-34 (EPIC212110888, 08:30:18.91 $+22: 14: 09.3$ ) belongs to field-of-view 5, photometrically monitored by K2 between April 27th and July 10th, 2015. The data was reduced using both the Warwick (Armstrong et al. 2015a) and the LAM- K2 (Barros et al. 2015) pipelines. The detrended data (see Tables 1 and 2) show $1.9 \%$ and $0.8 \%$ dimmings every 4.099 and 2.996 days for K2-30 and K2-34, respectively (see Figs. 1 and 2).

\subsection{High spatial resolution imaging}

We obtained a high spatial resolution image of K2-34 and K2-30 with the instrument AstraLux at the $2.2 \mathrm{~m}$ telescope in Calar Alto Observatory (Spain). We used the lucky-imaging technique, obtaining 90000 frames (45000 frames), each with an exposure time of $0.040 \mathrm{~s}(0.080 \mathrm{~s})$ for $\mathrm{K} 2-34(\mathrm{~K} 2-30)$. using the maximum gain setting. The images were reduced with the observatory pipeline, which performs basic reduction of the individual frames, selects the frames with the best Strehl ratios (Strehl 1902), aligns those frames, and combines them to provide a
Table 2. Normalized detrended K2 light curve of K2-34 (see Sect. 2.1).

\begin{tabular}{rcc}
\hline \hline BJD (days) & Flux & Flux unc \\
\hline 2457139.630908 & 0.999991 & 0.000042 \\
2457139.651340 & 0.999988 & 0.000042 \\
2457139.671772 & 0.999930 & 0.000042 \\
2457139.692204 & 0.999910 & 0.000042 \\
2457139.712636 & 1.000159 & 0.000042 \\
$\ldots$ & $\ldots$ & $\ldots$ \\
\hline
\end{tabular}

Notes. The complete version of this table is available at the CDS.
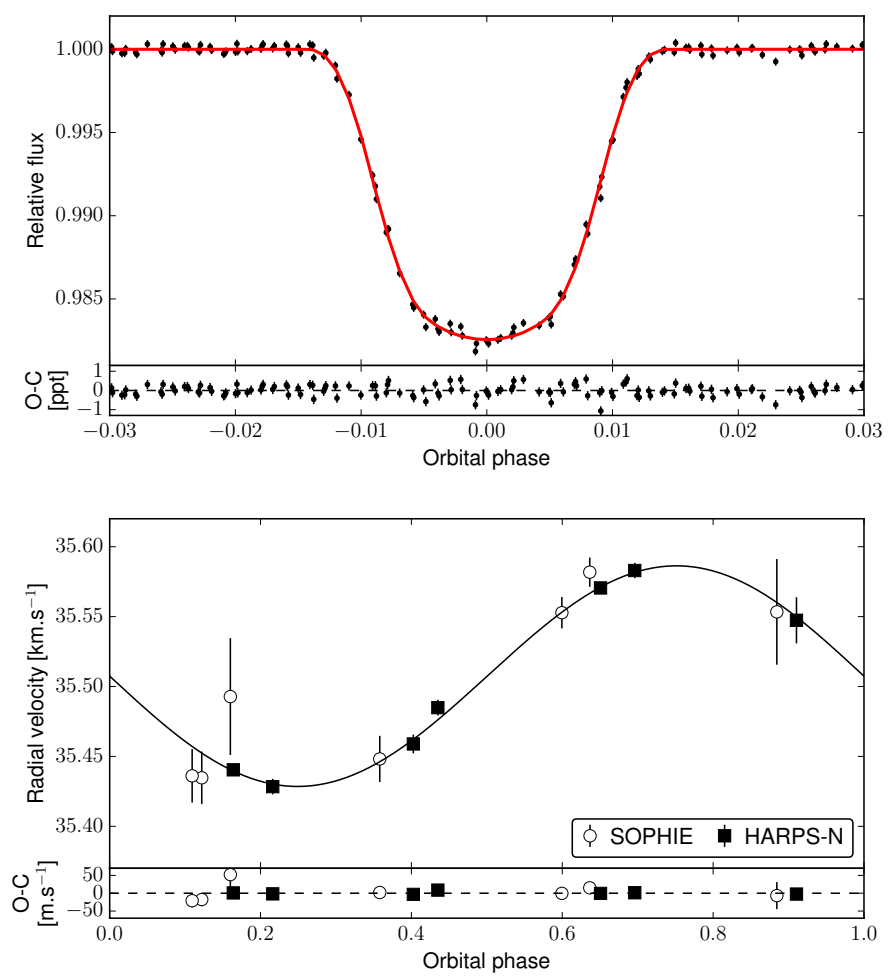

Fig. 1. Results of the joint analysis with PASTIS for K2-30, including the primary transit (top panel) and radial velocity (bottom panels). The final models are shown with solid lines and the residuals of the data are presented in the lower part of each panel.

final near-diffraction limited image. In this case, we selected the best $10 \%$ of the frames, which translates into an effective exposure time of $360 \mathrm{~s}$. No companion is detected within the sensitivity limits of the images. The sensitivity curve in each case was obtained by following the prescriptions in Lillo-Box et al. (2014a), simulating artificial stars at different positions in the reduced image and different contrast magnitudes and counting how many of them are recovered with a $5 \sigma$ signal-to-noise ratio. In summary, the image would allow us to detect companions with contrast magnitudes brighter than $3.5 \mathrm{mag}$ (3.7 mag) at $0.5 \mathrm{arcsec}, 5.3 \mathrm{mag}(5.0 \mathrm{mag})$ at $1 \mathrm{arcsec}$, and $8 \mathrm{mag}$ (6.0 mag) at 2 arcsec for K2-34 (K2-30). Since no companion is detected within these limits, we assume that $\mathrm{K} 2-34$ and $\mathrm{K} 2-30$ are isolated and that their light curves are not polluted by other sources. In Hirano et al. (2016), the authors found a faint companion to $\mathrm{K} 2-34$ at $361.3 \pm 3.5$ mas with $\Delta m_{\mathrm{H}}=6.19 \pm 0.11$. This source is below our detection limits, but owing to its faintness it has a negligible impact on the photometric analysis. The 

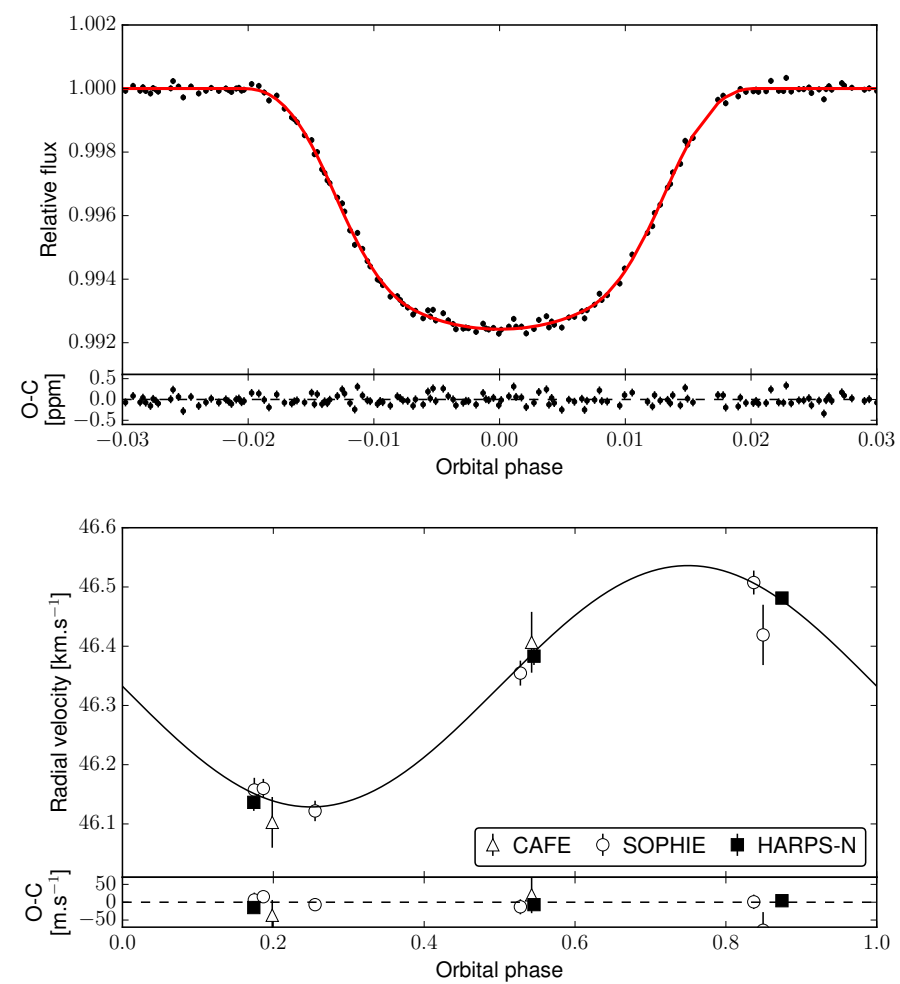

Fig. 2. Results of the joint analysis with PASTIS for K2-34. Same symbols as in Fig. 1.

maximum contrast for a blended eclipsing binary to be able to mimic the detected transit of K2-34 in the Kepler band would be $\Delta m_{\mathrm{Kep}}^{\max }=5.2 \mathrm{mag}$. Consequently, it is not possible that the close companion is the source of the eclipse. Hence, in practice, we can treat the system as being isolated.

\subsection{High-resolution spectroscopy}

We observed the two transited stars with HARPS-N (Cosentino et al. 2012) at the Telescopio Nazionale Galileo (TNG), Spain, and SOPHIE (Bouchy et al. 2013) at the Observatoire de Haute-Provence (OHP), France. Two additional epochs for K2-34 were obtained with CAFE (Aceituno et al. 2013) at the $2.2 \mathrm{~m}$ telescope of the Calar Alto Observatory (CAHA), Spain. The three instruments are fiber-fed highresolution echelle spectrographs with resolving powers of $R \sim 40000$ (SOPHIE in the high-efficiency mode), $R=110000$ (HARPS-N), and $R=63000$ (CAFE) and with no movable pieces. They are located in isolated chambers to improve their stability. SOPHIE and HARPS-N are stabilized in temperature and pressure, while these ambient conditions are simply monitored in the case of CAFE to check for possible RV drifts. In the three cases, the data was reduced with the corresponding online pipelines ${ }^{1}$. The RV is subsequently computed by determining the weighted cross-correlation function (CCF) between the spectra and a G2V binary mask ${ }^{2}$ (Baranne et al. 1996; Pepe et al. 2002). The SOPHIE data were corrected for the charge transfer inefficiency present in the charge-couple device of the instrument (Santerne et al. 2012). The RVs were also corrected for instrumental drifts using the RV standard star

\footnotetext{
1 For details on the CAFE pipeline see http://www. caha.es/CAHA/Instruments/CAFE/softw.html

2 In the case of CAFE, see Sect. 2.3 in Lillo-Box et al. (2015).
}

Table 3. Radial velocity data for K2-30.

\begin{tabular}{rcccc}
\hline \hline MBJD (days) & RV $\left(\mathrm{km} \mathrm{s}^{-1}\right)$ & BIS $(\mathrm{m} / \mathrm{s})$ & $F W H M\left(\mathrm{~km} \mathrm{~s}^{-1}\right)$ & Instrument \\
\hline 392.35938 & $35.5624 \pm 0.0043$ & $-39.8 \pm 6.5$ & $6.8842 \pm 0.0087$ & HARPS-N \\
392.57382 & $35.5504 \pm 0.0055$ & $-18.9 \pm 8.2$ & $6.8885 \pm 0.0109$ & HARPS-N \\
393.33790 & $35.5809 \pm 0.0066$ & $-3.2 \pm 10.0$ & $6.8780 \pm 0.0133$ & HARPS-N \\
393.47098 & $35.6069 \pm 0.0056$ & $-37.6 \pm 8.4$ & $6.8795 \pm 0.0111$ & HARPS-N \\
394.35486 & $35.6925 \pm 0.0042$ & $-32.3 \pm 6.4$ & $6.8701 \pm 0.0085$ & HARPS-N \\
394.54090 & $35.7050 \pm 0.0054$ & $-45.8 \pm 8.1$ & $6.8717 \pm 0.0108$ & HARPS-N \\
395.41956 & $35.6694 \pm 0.0165$ & $3.9 \pm 24.7$ & $6.8693 \pm 0.0329$ & HARPS-N \\
396.44205 & $35.493 \pm 0.042$ & $-24.5 \pm 75.1$ & $9.682 \pm 0.104$ & SOPHIE \\
398.39484 & $35.582 \pm 0.010$ & $-35.3 \pm 18.2$ & $9.523 \pm 0.025$ & SOPHIE \\
399.41136 & $35.554 \pm 0.038$ & $-18.1 \pm 67.9$ & $9.415 \pm 0.094$ & SOPHIE \\
400.38519 & $35.435 \pm 0.019$ & $-47.5 \pm 33.5$ & $9.517 \pm 0.046$ & SOPHIE \\
401.35244 & $35.448 \pm 0.016$ & $-22.0 \pm 29.3$ & $9.572 \pm 0.040$ & SOPHIE \\
402.34190 & $35.553 \pm 0.011$ & $-2.7 \pm 19.6$ & $9.540 \pm 0.027$ & SOPHIE \\
404.43149 & $35.436 \pm 0.019$ & $-11.0 \pm 34.0$ & $9.591 \pm 0.047$ & SOPHIE \\
\hline
\end{tabular}

Notes. MBJD = modified barycentric Julian date (BJD-2 457 000).

Table 4. Radial velocity data for K2-34.

\begin{tabular}{rcccc}
\hline \hline MBJD (days) & RV $\left(\mathrm{km} \mathrm{s}^{-1}\right)$ & BIS $(\mathrm{m} / \mathrm{s})$ & $F W H M\left(\mathrm{~km} \mathrm{~s}^{-1}\right)$ & Instrument \\
\hline 381.59795 & $45.863 \pm 0.040$ & $-70 \pm 65$ & $10.597 \pm 0.080$ & CAFE \\
382.62804 & $46.167 \pm 0.049$ & $-178 \pm 70$ & $10.841 \pm 0.110$ & CAFE \\
392.60905 & $46.5458 \pm 0.0034$ & $35.3 \pm 5.1$ & $9.5015 \pm 0.0069$ & HARPS-N \\
393.50729 & $46.2009 \pm 0.0133$ & $40.7 \pm 19.9$ & $9.4696 \pm 0.0266$ & HARPS-N \\
394.61995 & $46.4477 \pm 0.0138$ & $36.6 \pm 20.8$ & $9.4864 \pm 0.0277$ & HARPS-N \\
398.43529 & $46.508 \pm 0.018$ & $10 \pm 32$ & $11.381 \pm 0.044$ & SOPHIE \\
399.44751 & $46.157 \pm 0.018$ & $-17 \pm 32$ & $11.410 \pm 0.045$ & SOPHIE \\
399.68876 & $46.122 \pm 0.014$ & $51 \pm 25$ & $11.553 \pm 0.035$ & SOPHIE \\
403.49977 & $46.355 \pm 0.019$ & $-16 \pm 33$ & $11.319 \pm 0.046$ & SOPHIE \\
465.38755 & $46.160 \pm 0.013$ & $26 \pm 23$ & $11.400 \pm 0.032$ & SOPHIE \\
467.37227 & $46.419 \pm 0.050$ & $32 \pm 90$ & $11.252 \pm 0.125$ & SOPHIE \\
\hline
\end{tabular}

Notes. MBJD = modified barycentric Julian date (BJD-2 457 000).

HD 56124 observed during the same nights and following the prescriptions in Santerne et al. (2014). CAFE RVs were also corrected using observations of the same standard star during the nights.

For K2-30, seven epochs were obtained with HARPS-N during four consecutive nights (January 4-7, 2016) having a signalto-noise ratio $(\mathrm{S} / \mathrm{N})$ per pixel at $550 \mathrm{~nm}$ at the level of $15-30$, and five epochs were obtained with SOPHIE in the subsequent seven nights (January $8-13,2016$ ) with $\mathrm{S} / \mathrm{N}$ of $10-25$. This provides a time span of ten days for this 4-day period planet. For K2-34, we obtained three epochs with HARPS-N on January 4-7 2016 (S/N of 16-50), four epochs with SOPHIE on January 10-15 2016 (S/N of 27-40), and two epochs with CAFE on December 24-25 2015 (S/N of 11-13). This encompasses a 22-day time span for this $\sim 3$-day period planet. The derived RV values are shown in Tables 3 and 4 . In both cases, the radial velocity variations show no significant correlation with the bisector values, indicating that they do not originate from blended (undetected) stars. We calculated the projected rotational velocity of the star using the corresponding CCF following Boisse et al. (2010) (see Table 7).

\section{Results}

\subsection{Stellar properties}

The spectral analysis was performed on the HARPS-N data. For K2-30 the seven spectra were combined with the final spectrum 
Table 5. List of free parameters used in the PASTIS analysis of the light curves, radial velocities and SED with their associated prior.

\begin{tabular}{|c|c|c|}
\hline Parameter & K2-30 & K2-34 \\
\hline \multicolumn{3}{|l|}{ Orbital parameters } \\
\hline $\begin{array}{l}\text { Orbital period } P[\mathrm{~d}] \\
\text { Epoch of first transit } T_{0}\left[\mathrm{BJD}_{\mathrm{TDB}}\right]-2450000 \\
\text { Orbital eccentricity } e \\
\text { Argument of periastron } \omega\left[^{\circ}\right] \\
\text { Inclination } i\left[^{\circ}\right]\end{array}$ & $\begin{array}{c}\mathcal{N}\left(4.09803 ; 1 \times 10^{-3}\right) \\
\mathcal{N}(7063.826 ; 0.1) \\
\beta(0.867 ; 3.03) \\
\mathcal{U}(0 ; 360) \\
\mathcal{S}(70 ; 90) \\
\end{array}$ & $\begin{array}{c}\mathcal{N}\left(2.996 ; 1 \times 10^{-3}\right) \\
\mathcal{N}(7141.35 ; 0.1) \\
0(\text { fixed }) \\
- \\
\mathcal{S}(70 ; 90)\end{array}$ \\
\hline \multicolumn{3}{|l|}{ Planetary parameters } \\
\hline $\begin{array}{l}\text { Radial velocity amplitude } K[\mathrm{~m} / \mathrm{s}] \\
\text { Planet-to-star radius ratio } a / R_{\star}\end{array}$ & $\begin{array}{c}\mathcal{U}(0 ; 1000) \\
\mathcal{U}(0 ; 0.5)\end{array}$ & $\begin{array}{c}\mathcal{U}(0 ; 1000) \\
\mathcal{U}(0 ; 0.5)\end{array}$ \\
\hline \multicolumn{3}{|l|}{ Stellar parameters } \\
\hline $\begin{array}{l}\text { Effective temperature } T_{\text {eff }}[\mathrm{K}] \\
\text { Surface gravity } \log g\left[\mathrm{~g} / \mathrm{cm}^{2}\right] \\
\text { Iron abundance }[\mathrm{Fe} / \mathrm{H}][\mathrm{dex}] \\
\text { Reddening } E(B-V)[\mathrm{mag}] \\
\text { Systemic radial velocity } V_{\text {sys }}\left[\mathrm{km} \mathrm{s}^{-1}\right] \\
\text { Distance to Earth } d[\mathrm{pc}] \\
\text { Linear limb darkening coefficient ua } \\
\text { Quadratic limb darkening coefficient ub }\end{array}$ & $\begin{array}{c}\mathcal{N}(5573 ; 38) \\
\mathcal{N}(4.32 ; 0.20) \\
\mathcal{N}(0.14 ; 0.03) \\
\mathcal{U}(0 ; 1) \\
\mathcal{U}(-100,100) \\
\mathcal{P}(2 ; 10 ; 1000) \\
\mathcal{U}(0,1.2) \\
\mathcal{U}(0,1.2)\end{array}$ & $\begin{array}{c}\mathcal{N}(6139 ; 50) \\
\mathcal{N}(4.07 ; 0.21) \\
\mathcal{N}(0.11 ; 0.04) \\
\mathcal{U}(0 ; 1) \\
\mathcal{U}(-100,100) \\
\mathcal{P}(2 ; 10 ; 1000) \\
\mathcal{U}(0,1.2) \\
\mathcal{U}(0,1.2)\end{array}$ \\
\hline \multicolumn{3}{|l|}{ Instrumental parameters } \\
\hline $\begin{array}{l}\text { CAFE radial velocity jitter } \sigma_{\mathrm{RV}, \mathrm{CAFE}}[\mathrm{m} / \mathrm{s}] \\
\text { SOPHIE radial velocity jitter } \sigma_{\mathrm{RV}, \mathrm{SOPHI}}[\mathrm{m} / \mathrm{s}] \\
\text { HARPS-N radial velocity jitter } \sigma_{\mathrm{RV}, \mathrm{HARPS}-\mathrm{N}}[\mathrm{m} / \mathrm{s}] \\
\text { CAFE - SOPHIE radial velocity offset } \Delta \mathrm{RV} \mathrm{S}_{\mathrm{S}, \mathrm{CAFE}}[\mathrm{m} / \mathrm{s}] \\
\text { HARPS-N - SOPHIE radial velocity offset } \Delta \mathrm{RV} \text { S, H-N }[\mathrm{m} / \mathrm{s}] \\
\text { SED jitter } \sigma_{\mathrm{SED}}[\mathrm{mag}]\end{array}$ & $\begin{array}{c}- \\
\mathcal{U}(0 ; 100) \\
\mathcal{U}(0 ; 100) \\
\mathcal{U}(-1000 ; 1000) \\
\mathcal{U}(-1000 ; 1000) \\
\mathcal{U}(0 ; 1)\end{array}$ & $\begin{array}{c}\mathcal{U}(0 ; 100) \\
\mathcal{U}(0 ; 100) \\
\mathcal{U}(0 ; 100) \\
\mathcal{U}(-1000 ; 1000) \\
\mathcal{U}(-1000 ; 1000) \\
\mathcal{U}(0 ; 1)\end{array}$ \\
\hline
\end{tabular}

Notes. $\mathcal{N}\left(\mu ; \sigma^{2}\right)$ is a normal distribution with mean $\mu$ and width $\sigma^{2}, \mathcal{U}(a ; b)$ is a uniform distribution between $a$ and $b, \mathcal{S}(a ; b)$ is a sine distribution between $a$ and $b, \beta(a ; b)$ is a Beta distribution with parameters $a$ and $b$, and $\mathcal{P}(n ; a ; b)$ is a power-law distribution of exponent $n$ between $a$ and $b$.

References. The choice of prior for the orbital eccentricity is described in Kipping (2013).

reaching a $\mathrm{S} / \mathrm{N}$ of 56 . In the case of $\mathrm{K} 2-34$, we used a single spectrum with $\mathrm{S} / \mathrm{N}$ of 50 . The spectroscopic parameters were derived with the ARES+MOOG method (see Sousa 2014, for details) which is based on the measurement of equivalent widths of iron lines with ARES (Sousa et al. 2015). This method has been used to derive homogeneous parameters for planet-host stars (e.g., Santos et al. 2013). The derived properties ( $T_{\text {eff }}, \log g$, and $[\mathrm{Fe} / \mathrm{H}])$ for these G8V (K2-30) and F9V (K2-34) stars were used as priors for the joint analysis of the data (see Sect. 3.2) and are provided in Table 5. We also determined the lithium abundance for these host stars. We found an abundance of $A(\mathrm{Li})=$ $2.16 \pm 0.2$ dex for $\mathrm{K} 2-34\left(T_{\text {eff }}=6130 \pm 50 \mathrm{~K}\right)$ and an upper limit of $\mathrm{A}(\mathrm{Li})<0.9$ dex for $\mathrm{K} 2-30\left(T_{\text {eff }}=5585 \pm 38 \mathrm{~K}\right)$. These abundances provide an estimated lower limit for the age of both targets of 2 Gyr when compared to the abundances of members of the NGC 752 (Sestito et al. 2004) or M67 cluster (Pasquini et al. 2008).

\subsection{Joint analysis of the data}

We used the PASTIS software (Díaz et al. 2014; Santerne et al. 2015) to perform a joint analysis of the K2 light curve, radial velocities, and magnitudes of the two targets. The transit signals were modeled ${ }^{3}$ using a modified version of the JKTEBOP code (Southworth 2011, and references therein) and a Keplerian orbit was fitted to the RV data. The spectral energy distribution (SED, Table 6) was modeled with the BT-SETTL library (Allard 2014) and the stellar parameters were derived using the Dartmouth stellar evolution tracks (Dotter et al. 2008).

We performed a statistical analysis using Markov chain Monte Carlo (MCMC) algorithms. The model is described by six free parameters for the host star $\left(T_{\text {eff }}, \log g,[\mathrm{Fe} / \mathrm{H}]\right.$, systemic radial velocity $V_{\text {sys }}$, interstellar extinction $E(B-V)$, and distance $d$ ) and seven free parameters for the transit (period $P$, epoch of first transit $T_{0}$, radial velocity amplitude $K$, radius ratio $R_{p} / R_{\star}$, orbital eccentricity $e$, inclination $i$, and argument of periastron $\omega$ ). For the light curve, we also fit for an additional source of white noise, the out-of-transit flux level, and the level of contamination. For the RV, we additionally fit for a jitter term for each instrument and a RV offset between SOPHIE and the other instruments used. A jitter is also added for the SED analysis. For K2-34, owing to the low number of RV data points, we assumed

\footnotetext{
3 Models are numerically integrated over the Kepler exposure time with an oversampling factor of 10 .
} 
Table 6. Photometry used for the spectral energy distribution analysis of K2-30 and K2-34.

\begin{tabular}{rcc}
\hline \hline Band & K2-30 & K2-34 \\
\hline Johnson- $V$ & $13.53 \pm 0.039$ & $11.548 \pm 0.057$ \\
Johnson- $B$ & $14.506 \pm 0.030$ & $12.429 \pm 0.033$ \\
$g^{\prime}$ & - & $11.892 \pm 0.119$ \\
$r^{\prime}$ & $13.184 \pm 0.042$ & $11.389 \pm 0.026$ \\
$i^{\prime}$ & $12.819 \pm 0.046$ & $11.264 \pm 0.038$ \\
2MASS- $J$ & $11.632 \pm 0.019$ & $10.528 \pm 0.025$ \\
2MASS- $H$ & $11.190 \pm 0.016$ & $10.258 \pm 0.022$ \\
2MASS- $K$ s & $11.088 \pm 0.020$ & $10.193 \pm 0.017$ \\
WISE- $W 1$ & $11.016 \pm 0.023$ & $10.174 \pm 0.023$ \\
WISE- $W 2$ & $11.058 \pm 0.021$ & $10.207 \pm 0.020$ \\
WISE- $W 3$ & $11.067 \pm 0.161$ & $10.159 \pm 0.169$ \\
\hline
\end{tabular}

Notes. Optical magnitudes (Johnson- $V$, Johnson- $B, g^{\prime}, r^{\prime}$, and $i^{\prime}$ ) were obtained from the APASS database. Infrared values were obtained from Cutri et al. (2013).

a circular orbit ${ }^{4}$. This assumption is justified by the short period and circularization mechanisms. In total, 20 free parameters are fitted for both systems. Uniform priors are used for all free parameters except for the stellar values that were constrained to the results of the spectral analysis (see Sect. 3.1). The list of priors is shown in Table 5. We ran 20 chains of $3 \times 10^{5}$ iterations randomly drawn from the joint prior distribution. All chains converged toward the same solution, which is assumed to be the global maximum. In order to obtain the final solution and its uncertainties, we removed the burn-in phase of each chain and thinned them by computing their maximum correlation length. At this point we merged all of them to compute a well-sampled and clean posterior distribution having more than 2000 independent samples. The median values are presented in Table 7 together with the 15.7th and 84.3th percentiles of the marginalized distributions. In the table we also present other parameters derived from those fitted by the model (planet mass, planet radius, stellar mass, etc.). The data and best fit models are presented in Figs. 1, 2, and 4.

\section{Discussion}

The combination of different datasets for K2-30 b and K2-34 b establishes the planetary nature of the transiting objects around two main-sequence stars (G8V and F9V, respectively) observed by $\mathrm{K} 2$. The analysis of the data indicates that both systems are composed of single giant planets $\left(1.197 \pm 0.052 R_{\text {Jup }}\right.$ for K2-30 and $1.217 \pm 0.053 R_{\text {Jup }}$ for $\left.\mathrm{K} 2-34\right)$ in close-in orbits around their main-sequence stars.

In the case of K2-30, the insolation flux received by the planet is $F=4.24 \pm 0.37 \times 10^{8} \mathrm{erg} \mathrm{s}^{-1} \mathrm{~cm}^{-2}$. This is significantly larger than the empirical cut-off limit derived by Demory \& Seager (2011) for a planet to have an inflated radius $\left(F>2.08 \times 10^{8} \mathrm{erg} \mathrm{s}^{-1} \mathrm{~cm}^{-2}\right)$. Assuming a Bond albedo $A=0$ and a complete redistribution of the tidal heating along the planet, its expected radius according to Eq. (9) in

\footnotetext{
4 We tested the non-circular hypothesis and compared the Bayesian information criterion (BIC; see, e.g., Schwarz 1978; Smith et al. 2009) of the two models. As expected, the results provide strong evidence for the circular model given the current data, with a BIC difference of 2 in favor of the circular (i.e., simpler) hypothesis.
}

Table 7. Host star, planet, and orbital parameters inferred from the joint analysis of the data.

\begin{tabular}{|c|c|c|}
\hline Parameter & $\mathrm{K} 3-30$ & $\mathrm{~K} 2-34$ \\
\hline \multicolumn{3}{|l|}{ Planet properties } \\
\hline$K(\mathrm{~m} / \mathrm{s})$ & $80.1 \pm 3.8$ & $205 \pm 12$ \\
\hline$R_{p} / R_{\star}$ & $0.1303 \pm 0.0025$ & $0.0901 \pm 0.0018$ \\
\hline$M_{p}\left(M_{\mathrm{Jup}}\right)$ & $0.625 \pm 0.030$ & $1.649 \pm 0.098$ \\
\hline$R_{p}\left(R_{\mathrm{Jup}}\right)$ & $1.197 \pm 0.052$ & $1.217 \pm 0.053$ \\
\hline$\rho_{p}\left(\rho_{\text {Jup }}\right)$ & $0.363 \pm 0.055$ & $0.92 \pm 0.14$ \\
\hline$T_{\mathrm{eq}}(\mathrm{K})$ & $1152 \pm 19$ & $1670 \pm 26$ \\
\hline \multicolumn{3}{|l|}{ Orbital properties } \\
\hline Period (day) & $4.098513 \pm 0.000018$ & $2.9956675_{-0.000071}^{+0.0000075}$ \\
\hline$T_{0}\left(\mathrm{MBJD}^{a}\right.$, day $)$ & $63.80699 \pm 0.00017$ & $141.35103_{-0.00018}^{+0.00068}$ \\
\hline$i(\operatorname{deg})$ & $86.34 \pm 0.35$ & $83.03 \pm 0.37$ \\
\hline$e$ & $0.026_{-0.020}^{+0.038}$ & 0.0 (assumed) \\
\hline$\omega(\mathrm{deg})$ & $115_{-48}^{+100}$ & - \\
\hline$a(\mathrm{au})$ & $0.04986 \pm 0.00033$ & $0.04328 \pm 0.00037$ \\
\hline$a / R_{\star}$ & $11.40 \pm 0.48$ & $6.73 \pm 0.21$ \\
\hline$b_{\text {primary }}$ & $0.714 \pm 0.029$ & $0.816 \pm 0.018$ \\
\hline$T_{\text {dur }}(\mathrm{h})$ & $2.362 \pm 0.021$ & $2.463 \pm 0.015$ \\
\hline \multicolumn{3}{|l|}{ Host properties } \\
\hline$M_{\star}\left(M_{\odot}\right)$ & $0.984 \pm 0.020$ & $1.204 \pm 0.031$ \\
\hline$R_{\star}\left(R_{\odot}\right)$ & $0.941 \pm 0.038$ & $1.383 \pm 0.046$ \\
\hline$\rho_{\star}\left(\rho_{\odot}\right)$ & $1.18 \pm 0.15$ & $0.356 \pm 0.044$ \\
\hline$V_{\text {sys }}\left(\mathrm{km} \mathrm{s}^{-1}\right)$ & $35.5092 \pm 0.0088$ & $46.330 \pm 0.013$ \\
\hline$d(\mathrm{pc})$ & $323 \pm 13$ & $341 \pm 12$ \\
\hline$E(B-V)$ & $0.253 \pm 0.014$ & $0.038 \pm 0.020$ \\
\hline $\log g(\operatorname{cgs})$ & $4.484 \pm 0.039$ & $4.236 \pm 0.025$ \\
\hline$T_{\text {eff }}$ & $5582 \pm 39$ & $6131 \pm 47$ \\
\hline$[\mathrm{Fe} / \mathrm{H}](\mathrm{dex})$ & $0.137 \pm 0.031$ & $0.121 \pm 0.053$ \\
\hline $\log L / L_{\odot}(\operatorname{dex})$ & $-0.112 \pm 0.039$ & $0.471 \pm 0.048$ \\
\hline ua & $0.24 \pm 0.20$ & $0.23 \pm 0.23$ \\
\hline $\mathrm{ub}$ & $0.41 \pm 0.29$ & $0.46 \pm 0.28$ \\
\hline$v \sin i\left(\mathrm{~km} \mathrm{~s}^{-1}\right)$ & $3.9 \pm 1.1$ & $5.2 \pm 1.4$ \\
\hline \multicolumn{3}{|c|}{ Jitters $(\sigma)$ and instrumental offsets $(\Delta \mathrm{RV})$} \\
\hline$\sigma_{\mathrm{RV}, \mathrm{HARPS}-\mathrm{N}}(\mathrm{m} / \mathrm{s})$ & $3.4_{-2.4}^{+4.9}$ & $18_{13}^{+28}$ \\
\hline$\sigma_{\mathrm{RV}, \mathrm{SOPHIE}}(\mathrm{m} / \mathrm{s})$ & $10.7_{-7.3}^{+13.0}$ & $15_{-11}^{+20}$ \\
\hline$\sigma_{\mathrm{RV}, \mathrm{CAFE}}(\mathrm{m} / \mathrm{s})$ & - & $43 \pm 35$ \\
\hline$\Delta \mathrm{RV}_{\mathrm{S}, \mathrm{H}-\mathrm{N}}(\mathrm{m} / \mathrm{s})$ & $-121.5 \pm 9.0$ & $-64 \pm 21$ \\
\hline$\Delta \mathrm{RV}_{\mathrm{S}, \mathrm{CAFE}}(\mathrm{m} / \mathrm{s})$ & - & $246 \pm 48$ \\
\hline$\sigma_{\mathrm{K} 2}(\mathrm{ppm})$ & $221 \pm 12$ & $87.2 \pm 3.9$ \\
\hline$\sigma_{\mathrm{SED}}(\mathrm{mag})$ & $0.017 \pm+0.016$ & $0.071_{-0.020}^{+0.029}$ \\
\hline $\mathrm{K} 2$ contamination & $0.030_{-0.021}^{+0.036}$ & $0.033_{-0.023}^{+0.039}$ \\
\hline
\end{tabular}

Notes. Uncertainties represent the $15.7 \%$ and $84.3 \%$ percentiles of the marginalized posterior distribution. ${ }^{(a)}$ Modified barycentric Julian date $=$ BJD-2 457000 (days).

Enoch et al. (2012) would be $0.777 \pm 0.014 R_{\text {Jup }}{ }^{5}$. According to Weiss et al. (2013) the mass-radius-insolation flux relation provides an expected radius for this planet of $1.151 \pm 0.010 R_{\text {Jup }}$. In the case of $\mathrm{K} 2-34 \mathrm{~b}$, the planet receives an insolation flux

5 Larger albedos would imply smaller expected planetary radius, so this can be considered as an upper limit. 


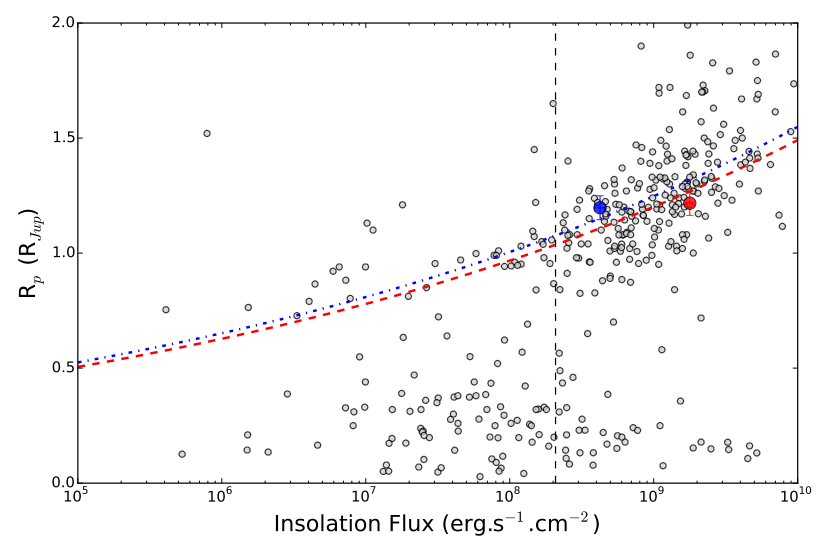

Fig. 3. Insolation flux and planet radius for all extrasolar planets with known radius, $T_{\text {eff }}$, and semi-major axis. Data taken from the The Extrasolar Planet Encyclopedia. The two hot Jupiters found in this work are marked as filled circles (K2-34 in red and K2-30 in blue). The expected radius-insolation flux dependencies according to Weiss et al. (2013) are shown as dashed red (K2-34) and dash-dotted blue (K2-30) lines.

of $F=1.768 \pm 0.14 \times 10^{9} \mathrm{erg} \mathrm{s}^{-1} \mathrm{~cm}^{-2}$. This is one order of magnitude larger than the above-mentioned cut-off limit derived by Demory \& Seager (2011). The expected radius according to Enoch et al. (2012) would be $0.993 \pm 0.016 R_{\text {Jup }}$ and the expected radius from Weiss et al. (2013) is $1.267 \pm 0.010 R_{\mathrm{Jup}}$.

In both cases, the planets have comparable radii to those predicted by empirical calibrations (see Fig. 3). Although compared to the values predicted by Weiss et al. (2013) they are compatible within $1 \sigma$ with being inflated due to the high stellar insolation flux, both are clearly larger $(>3 \sigma)$ than the predicted value by Enoch et al. (2012). It is known that high stellar irradiance can explain the inflation of the close-in Jupiter planets with radii up to $\sim 1.2 R_{\text {Jup }}$ (Guillot \& Showman 2002). However, this cannot explain larger radii, and so other mechanisms must play a role (e.g., Bodenheimer et al. 2001; Batygin \& Stevenson 2010; Chabrier \& Baraffe 2007). The small eccentricity found in K2-30 (but statistically not significant) could possibly indicate some tidal heating, but other mechanisms cannot be rejected.

In this paper, we have characterized two HJs. They show bloated radii possibly due to the large stellar insolation that they are receiving from their host. However, other possible mechanisms such as tidal heating may be playing a role. Since the hosts are bright ( $V=11.5$ for $\mathrm{K} 2-34$ and $V=13.5$ for $\mathrm{K} 2-30)$ and the planets are inflated, they are amenable for atmospheric characterization either from the ground or from space. As they transit, they are also good candidates for the detection of the Rossiter-MacLaughlin effect used to infer the spin-orbit angle and to study the evolutionary history of these systems. From the derived parameters, the amplitude of this effect should be around $60 \mathrm{~m} / \mathrm{s}$ and $40 \mathrm{~m} / \mathrm{s}$ for K2-30 and K2-34, respectively. Future searches for additional bodies will be interesting in order to unveil planet-planet interactions during the onset of the planetary systems.

The K2-34 system was also analyzed by Hirano et al. (2016), with good agreement in the parameters directly derived from modeling the observations. Owing to small differences in the determined stellar properties, some absolute physical and orbital parameters disagree by a small percentage. Johnson et al. (2016) also analyzed K2-30 and their results are mainly in agreement with ours. Some parameters are different by a small percentage, but this could be due to their assumption of a circular orbit and to
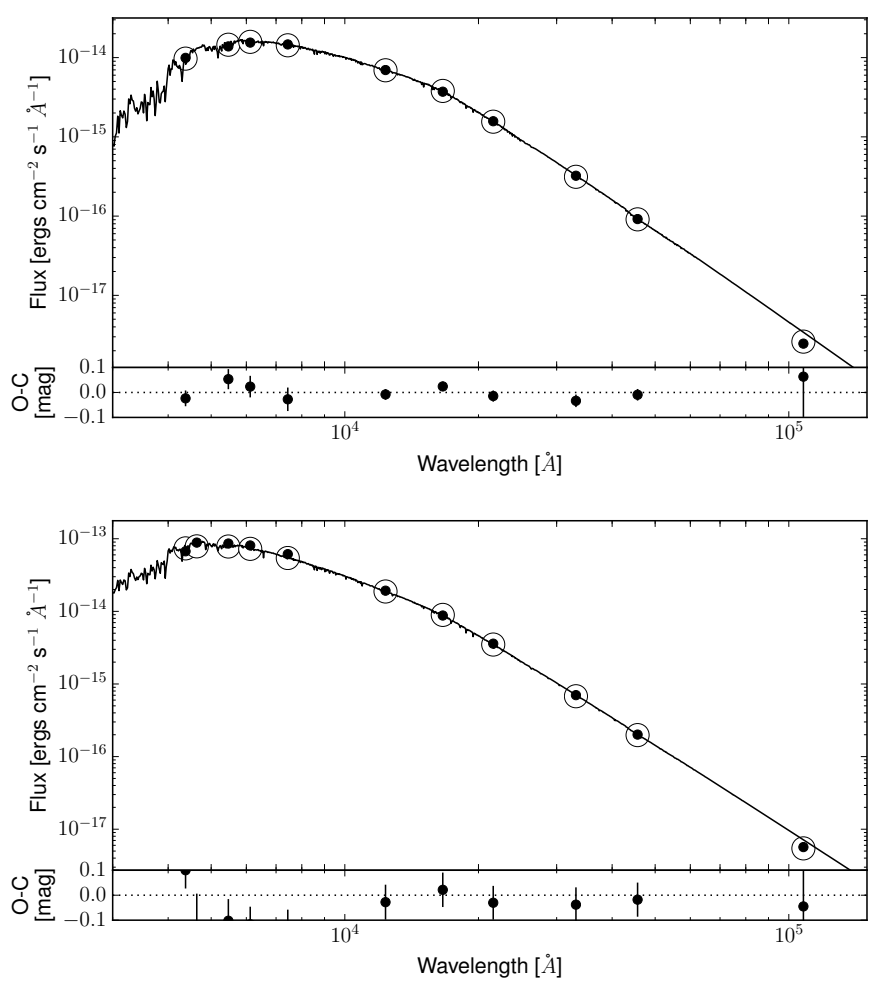

Fig. 4. Results of the SED fitting in the joint analysis of the data with PASTIS for K2-30 (top panel) and K2-34 (bottom panel). The final models are shown with solid lines and the residuals of the data are presented in the lower part of each panel.

their use of a different approach for the calculation of the stellar parameters.

Acknowledgements. J.L.-B. acknowledges financial support from the Marie Curie Actions of the European Commission (FP7-COFUND) and the Spanish grant AYA2012- 38897-C02-01. O.D. acknowledges support by CNES through contract 567133. A.S. is supported by the European Union under a Marie Curie Intra-European Fellowship for Career Development with reference FP7-PEOPLE-2013-IEF, number 627202. D.J.A. and D.P. acknowledge funding from the European Union Seventh Framework programme (FP7/20072013) under grant agreement No. 313014 (ETAEARTH). J.-M.A. acknowledges funding from the European Research Council under the ERC Grant Agreement n. 337591-ExTrA. P.A.W acknowledges the support of the French Agence Nationale de la Recherche (ANR), under program ANR-12-BS050012 "Exo-Atmos". A.S., S.C.C.B., E.D.M., N.C.S., S.S., and M.T. acknowledge support by Fundação para a Ciência e a Tecnologia (FCT) through the research grants UID/FIS/04434/2013 (POCI-01-0145-FEDER-007672) and project PTDC/FIS-AST/1526/2014. N.C.S., S.G.S., and S.C.C.B. acknowledge the support from FCT through Investigador FCT contracts of reference IF/00169/2012, IF/00028/2014, and IF/01312/2014, respectively, and POPH/FSE (EC) by FEDER funding through the program "Programa Operacional de Factores de Competitividade - COMPETE". E.D.M. acknowledges the support from FCT in the form of the grant SFRH/BPD/76606/2011. This publication is based on observations collected with the NASA satellite Kepler, with the SOPHIE spectrograph at OHP (CNRS, France), HARPS-N (La Palma, Spain), and CAFE and AstraLux at Calar Alto Observatory (Spain). This publication makes use of data products from the Wide-field Infrared Survey Explorer, which is a joint project (UCLA/JPL) funded by NASA. This research made use of the AAVSO Photometric All-Sky Survey, funded by the Robert Martin Ayers Sciences Fund.

\section{References}

Aceituno, J., Sánchez, S. F., Grupp, F., et al. 2013, A\&A, 552, A31 Adams, E. R., Ciardi, D. R., Dupree, A. K., et al. 2012, AJ, 144, 42 Allard, F. 2014, in IAU Symp. 299, eds. M. Booth, B. C. Matthews, \& J. R. Graham, 271 
Armitage, P. J. 2003, ApJ, 582, L47

Armstrong, D. J., Kirk, J., Lam, K. W. F., et al. 2015a, A\&A, 579, A19

Armstrong, D. J., Santerne, A., Veras, D., et al. 2015b, A\&A, 582, A33

Baranne, A., Queloz, D., Mayor, M., et al. 1996, A\&AS, 119, 373

Barros, S. C. C., Almenara, J. M., Demangeon, O., et al. 2015, MNRAS, 454, 4267

Batygin, K., \& Stevenson, D. J. 2010, ApJ, 714, L238

Becker, J. C., Vanderburg, A., Adams, F. C., Rappaport, S. A., \& Schwengeler, H. M. 2015, ApJ, 812, L18

Bodenheimer, P., Lin, D. N. C., \& Mardling, R. A. 2001, ApJ, 548, 466

Boisse, I., Eggenberger, A., Santos, N. C., et al. 2010, A\&A, 523, A88

Bouchy, F., Díaz, R. F., Hébrard, G., et al. 2013, A\&A, 549, A49

Chabrier, G., \& Baraffe, I. 2007, ApJ, 661, L81

Cosentino, R., Lovis, C., Pepe, F., et al. 2012, in SPIE Conf. Ser., 8446, 1

Crossfield, I. J. M., Petigura, E., Schlieder, J. E., et al. 2015, ApJ, 804, 10

Cutri, R. M., \& et al. 2013, VizieR Online Data Catalog: II/328

Demory, B.-O., \& Seager, S. 2011, ApJS, 197, 12

Díaz, R. F., Almenara, J. M., Santerne, A., et al. 2014, MNRAS, 441, 983

Dotter, A., Chaboyer, B., Jevremović, D., et al. 2008, ApJS, 178, 89

Enoch, B., Collier Cameron, A., \& Horne, K. 2012, A\&A, 540, A99

Foreman-Mackey, D., Montet, B. T., Hogg, D. W., et al. 2015, ApJ, 806, 215

Guillot, T., \& Showman, A. P. 2002, A\&A, 385, 156

Hirano, T., Nowak, G., Kuzuhara, M., et al. 2016, ApJ, 825, 53

Howell, S. B., Sobeck, C., Haas, M., et al. 2014, PASP, 126, 398

Johnson, M. C., Gandolfi, D., Fridlund, M., et al. 2016, AJ, 151, 171

Kipping, D. M. 2013, MNRAS, 434, L51

Law, N. M., Morton, T., Baranec, C., et al. 2014, ApJ, 791, 35

Lillo-Box, J., Barrado, D., \& Bouy, H. 2012, A\&A, 546, A10

Lillo-Box, J., Barrado, D., Moya, A., et al. 2014a, A\&A, 562, A109
Lillo-Box, J., Barrado, D., \& Bouy, H. 2014b, A\&A, 566, A103

Lillo-Box, J., Barrado, D., Santos, N. C., et al. 2015, A\&A, 577, A105

Morton, T. D., \& Johnson, J. A. 2011, ApJ, 729, 138

Pasquini, L., Biazzo, K., Bonifacio, P., Randich, S., \& Bedin, L. R. 2008, A\&A, 489, 677

Pepe, F., Mayor, M., Galland, F., et al. 2002, A\&A, 388, 632

Petigura, E. A., Schlieder, J. E., Crossfield, I. J. M., et al. 2015, ApJ, 811, 102

Sanchis-Ojeda, R., Rappaport, S., Pallè, E., et al. 2015, ApJ, 812, 112

Santerne, A., Díaz, R. F., Moutou, C., et al. 2012, A\&A, 545, A76

Santerne, A., Hébrard, G., Deleuil, M., et al. 2014, A\&A, 571, A37

Santerne, A., Díaz, R. F., Almenara, J.-M., et al. 2015, MNRAS, 451, 2337

Santerne, A., Moutou, C., Tsantaki, M., et al. 2016, A\&A, 587, A64

Santos, N. C., Sousa, S. G., Mortier, A., et al. 2013, A\&A, 556, A150

Schwarz, G. 1978, Ann. Stat., 6, 461

Sestito, P., Randich, S., \& Pallavicini, R. 2004, A\&A, 426, 809

Showman, A. P., \& Guillot, T. 2002, A\&A, 385, 166

Smith, R., Churcher, L. J., Wyatt, M. C., Moerchen, M. M., \& Telesco, C. M. 2009, A\&A, 493, 299

Sousa, S. G. 2014, in Determination of Atmospheric Parameters of B-, A-, Fand G-Type Stars (Springer), 297

Sousa, S. G., Santos, N. C., Adibekyan, V., Delgado-Mena, E., \& Israelian, G. 2015, A\&A, 577, A67

Southworth, J. 2011, MNRAS, 417, 2166

Steffen, J. H., Ragozzine, D., Fabrycky, D. C., et al. 2012, Proc. Nat. Acad. Sci., 109, 7982

Strehl, K. 1902, Astron. Nachr., 158, 89

Weiss, L. M., Marcy, G. W., Rowe, J. F., et al. 2013, ApJ, 768, 14

Winn, J. N., Noyes, R. W., Holman, M. J., et al. 2005, ApJ, 631, 1215 